\title{
Combining in situ heating with transmission diffraction and imaging in SEM for investigation of early stages of solid-state dewetting
}

Peter Denninger ${ }^{1}$, Peter Schweizer ${ }^{2}$, Tim Schwope ${ }^{1}$, Christian Dolle ${ }^{3}$ and Erdmann Spiecker ${ }^{1}$

${ }^{1}$ Institute of Micro- and Nanostructure Research (IMN) \& Center for Nanoanalysis and Electron Microscopy (CENEM), Interdisciplinary Center for Nanostructured Films (IZNF), Department of Materials Science and Engineering, Friedrich-Alexander University Erlangen-Nürnberg (FAU), Erlangen, Germany, Erlangen, Bayern, Germany, ${ }^{2}$ Empa, Thun, Switzerland, ${ }^{3}$ Karlsruher Institut für Technologie, Karlsruhe, Baden-Wurttemberg, Germany

In recent years transmission electron microscopy (TEM) has moved to lower electron energies owing to aberration correction and improvement in instrumentation which allows to maintain high spatial resolution down to atomic scale. Depending on the material under investigation usage of smaller electron energies has several benfits including stronger contrast of light elements and suppression of knock-on damage. Scanning Electron Microscopy (SEM) already operates with lower beam energies (1-30 keV) and can partly take over TEM work in this energy range as long as atomic resolution is not required. Therefore, more and more imaging and diffraction techniques originally developed in TEM/STEM are transferred to SEM. Modern SEM instruments are typically equipped with a retractable solid-state detector enabling STEM imaging with down to sub-nanometer resolution. SEM also offers several diffraction techniques, such as Electron Backscatter Diffraction (EBSD) and Transmission Kikuchi Diffraction (TKD) but real on-axis transmission diffraction is still in development. Several concepts of transmission diffraction have been proposed and first setups have been established to combine the unique features of an SEM with diffraction analysis [1-3]. Among these unique features are the large sample chamber and the availability of additional imaging modes (SE, BSE) typically not available in TEM which together provide high flexibility and unique opportunities for in situ microscopy and multimodal imaging.

One simple solution for transmission diffraction in SEM is our successfully implemented Low Energy Nano Diffraction (LEND) setup [3], which is based on the combination of a fluorescent screen positioned below the sample with a dedicated CMOS camera (Figure 1a). LEND produces TEM-like nanodiffraction patterns containing rich information on the crystal structure of the sample. By combining LEND and STEM in SEM various material systems have been investigated, such as thin metal films and graphene. Furthermore, our setup supports 4D-STEM imaging [3], which offers unique data analysis, such as formation of virtual dark-field images as well as strain and orientation mapping.

Complex reactions and processes in materials are challenging to be investigated by ex situ experiments. Often, microscopic mechanisms cannot be unraveled based on single snapshots of the sample state. Especially for phase transitions and material transport at high temperatures continuous monitoring is essential [4]. Therefore, in situ heating experiments have been established itself as an indispensable tool in many areas of materials research and for different kinds of microscopy. An SEM offers also great possibilities for in situ experiments, such as low acceleration voltages, large field of view, large microscope chamber and strong topographic contrast. However, the combination of in situ heating with transmission imaging has not been accomplished so far.

In this work, we introduce an update of our LEND setup, which enables combined in situ heating, imaging, and transmission diffraction in SEM (Figure 1b-c). It is based on a custom-built heating stage for DENSsolutions Wildfire Nano-Chips enabling extremely fast heating and cooling rates. The setup has been implemented and applied to the investigation of thin film processes, where the simultaneous 
acquisition of real- and reciprocal space information is needed to elucidate phenomena such as crystallization, grain coarsening and texture formation.

To showcase the benefits of combining several imaging modes with diffraction, we will present solidstate dewetting experiments on metal thin films. This phenomenon occurs for thin layers below their melting temperature and describes the degradation into particles or droplets due to the reduction of surface energy. It is an important process to fabricate nanoparticles with desired size, shape and composition. On the other hand, solid-state dewetting also constitutes a degradation mechanism of thin films and has to be considered in applications to prevent device failure. In our earlier work we have already studied the process of solid-state dewetting for thin gold films using in situ chip-based heating combined with multimodal imaging and diffraction in TEM [5,6]. However, some questions related to solid-state dewetting cannot be analyzed purely by TEM due to the limited field of view and lack of topographic contrast

Using our novel in situ heating stage in SEM promises new insights into the interplay of crystallographic and topographic processes by combining imaging and diffraction modes. Figure 2a shows the initial state of the thin gold film with the corresponding polycrystalline ring diffraction pattern (Figure 2b). During heating the film shows dewetting accompanied by grain coarsening and texture evolution as nicely revealed by simultaneous in situ BSE imaging (Figure 2a,d) and in situ LEND (Figure 2b,e), respectively. A radial intensity profile of the acquired LEND patterns shows a reduction of intensity, especially for the (111) diffraction ring (Figure 2c), indicating evolution of a [111] texture [6]. The in situ experiment offers the great benefit of continuous and simultaneous monitoring of both, the diffraction peak intensities and the area coverage which are plotted as function of annealing time in Figure $2 \mathrm{f}$. Here the (220)/(111) intensity ratio is used to reveal the evolution of [111] texture and accomodate for increasing particle thickness. While dewetting and texture evolution occur largely simultaneously, there is a clear indication that texture formation slightly precedes the degradation/dewetting of the thin film. This interesting observation suggests that in the early stages of annealing competitive grain growth results in preferential growth of grains with [111] texture even before extensive material transport sets in. This key information could only be obtained by combining in situ imaging and diffraction in one experiment.

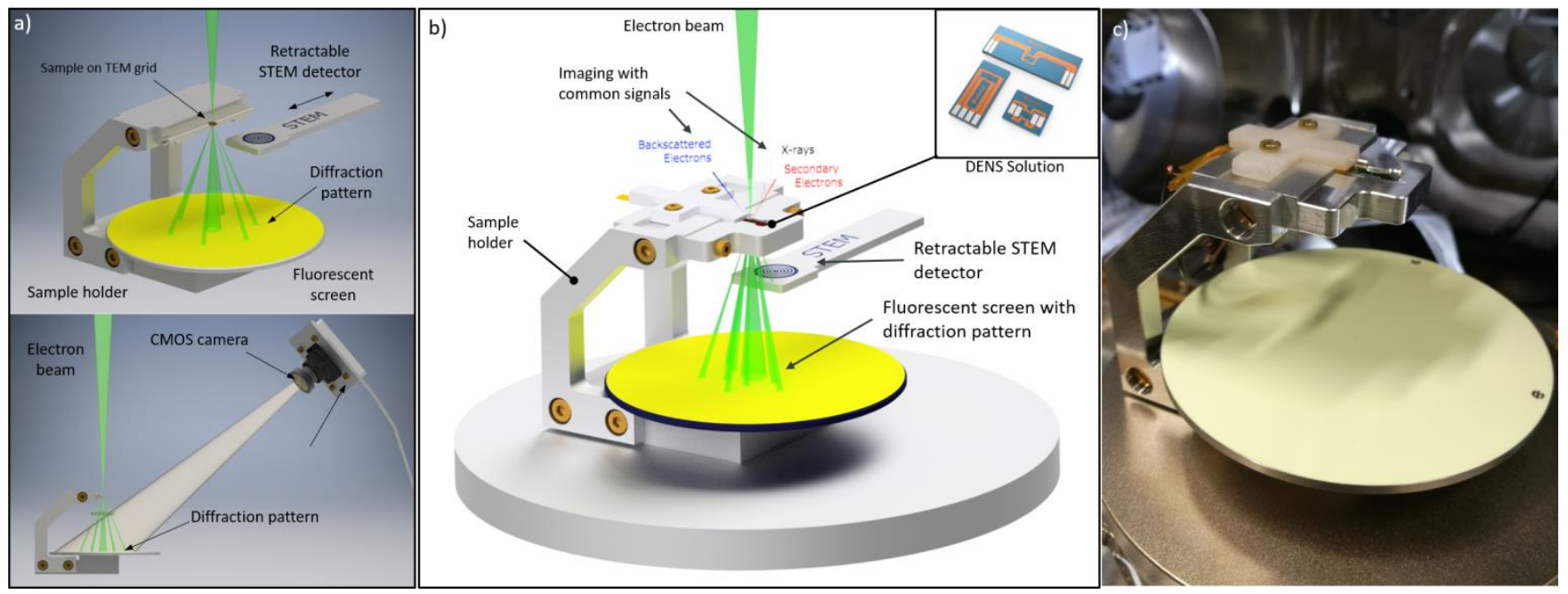

Figure 1. Figure 1: In situ heating and transmission diffraction setup in the SEM. a) Setup for Low Energy Nano Diffraction (LEND). b) Illustration of the developed heating stage for DENSsolutions heating chips. c) Installed heating stage in the SEM. 


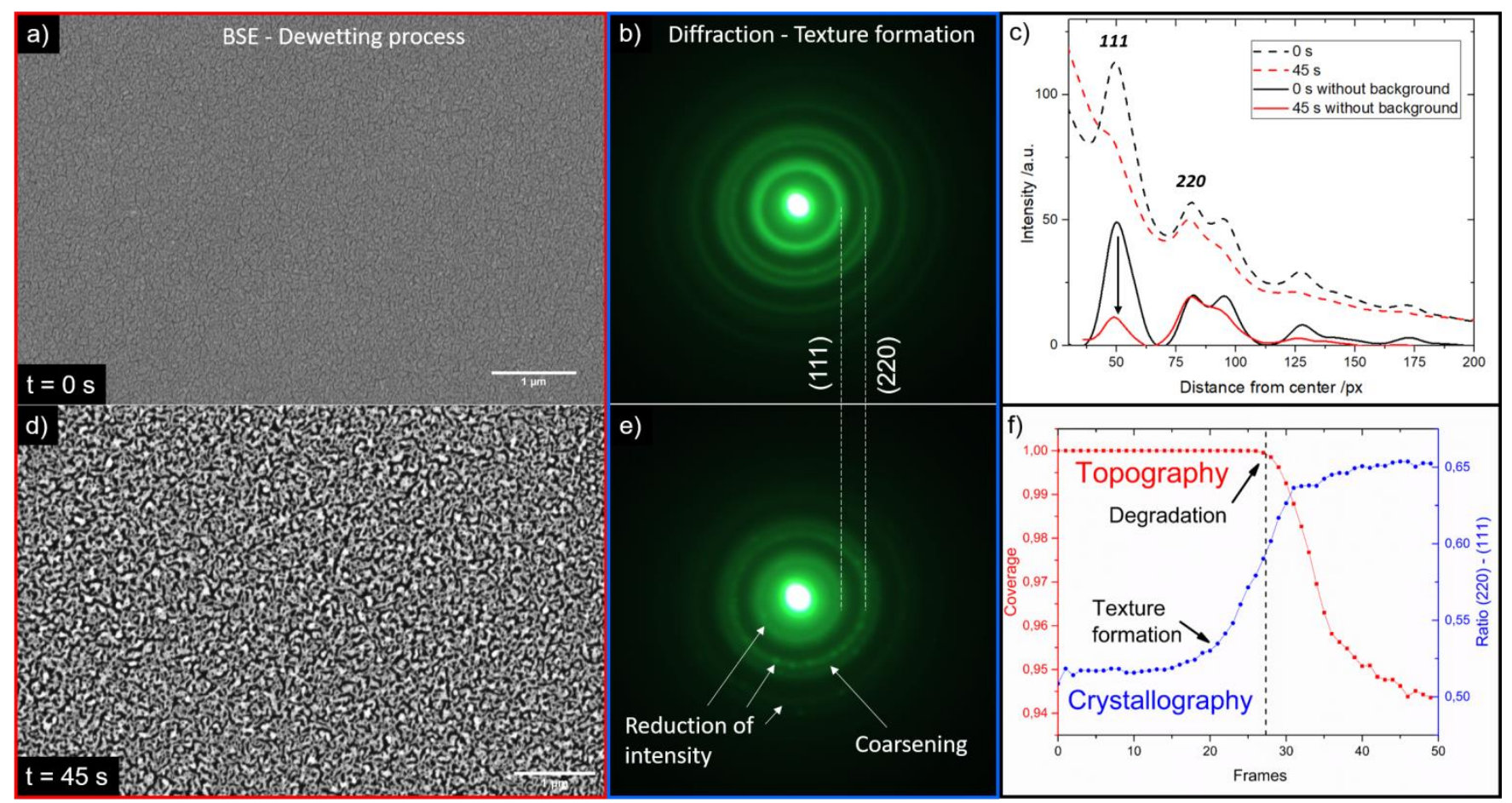

Figure 2. Figure 2: Investigation of solid-state dewetting of thin Au film. a) Initial state with corresponding poly-crystalline ring diffraction pattern (b). c) Radial intensity plot for both diffraction patterns support the observed reduction of intensity. d)-e) The final state displays degraded thin film, grain coarsening and texture evolution. f) Development of area coverage and (220)/(111) peak intensity ratio revealing initial texture formation before dewetting sets in.

\section{References}

[1] C. Sun, E. Muller, M. Meffert, D. Gerthsen, Analysis of crystal defects by scanning transmission electron microscopy (STEM) in a modern scanning electron microscope, Adv. Struct. Chem. Imaging 5 (2019) 1.

[2] B.W. Caplins, J.D. Holm, R.R. Keller, Transmission imaging with a programmable detector in a scanning electron microscope, Ultramicroscopy 196 (2019) 40-48.

[3] P. Schweizer, P. Denninger, C. Dolle, E. Spiecker, Low energy nano diffraction (LEND) - A versatile diffraction technique in SEM, Ultramicroscopy 213 (2020) 112956.

[4] G. Dehm, J. Howe, J. Zweck, In-situ Electron Microscopy: Applications in Physics, Chemistry and Materials Science, Wiley-VCH, Weinheim, 2012.

[5] F. Niekiel, P. Schweizer, S. M. Kraschewski, B. Butz, and E. Spiecker, The process of solid-state dewetting of Au thin films studied by in situ scanning transmission electron microscopy, Acta Materialia 90 (2015) 118132.

[6] F. Niekiel, S. M. Kraschewski, P. Schweizer, B. Butz, and E. Spiecker, Texture evolution and microstructural changes during solid-state dewetting: a correlative study by complementary in situ TEM techniques, Acta Materialia 115 (2016) 230-241.

[7] We gratefully acknowledge financial support by the German Research Foundation (DFG) within the frameworks of the research training group GRK1896 "In situ Microscopy with Electrons, X-rays and Scanning Probes" and the collaborative research centre SFB953 "Synthetic C-allotropes". 